\title{
“LO QUE PUEDEN ESTUDIAR LAS MUJERES EN VALPARAÍSO" DE GLADYS PEAKE. ANÁLISIS DE HABLA DE UN DOCUMENTO DE ÉPOCA
}

\section{“WHAT WOMEN CAN STUDY IN VALPARAÍSO” BY GLADYS PEAKE. ANALYSIS OF A PERIOD SPEECH DOCUMENT}

\author{
VALENTina Leal RomÁn ${ }^{*}$
}

\begin{abstract}
RESUMEN
En este artículo se analiza un documento del año 1945 (aproximadamente) que se titula "Lo que pueden estudiar las mujeres en Valparaíso", escrito por una de las primeras enfermeras del país Gladys Peake. Se lo examina por medio de un análisis de discurso, como un documento que representa una época determinada, que nos habla del contexto social, de la enfermería, sus características y visiones en un periodo dado. Los resultados muestran la prevalencia de un discurso propio de los orígenes de la enfermería profesional que necesitaba de un reconocimiento social, se argumenta por la necesidad de su valoración y se plantea la indispensabilidad de la enfermería en ese momento. A partir de allí se espera reflexionar sobre la profesión en el contexto actual.
\end{abstract}

Palabras clave: Historia de la enfermería, enfermería, habla.

\begin{abstract}
This paper analyzes a document entitled "What women can study in Valparaíso", written in 1945 (approximately) by one of the first nurses in Chile, Gladys Peake. This document is examined through a discourse analysis, as a document which depicts a particular age, which tells us about the nursing social context, its characteristics and visions at a certain period of time. Results show the prevalence of an own discourse referring the professional nursing origins as a method to obtain social awareness. It argue in favor of its assessment and the necessity of nursing in that period. From there we hope to reflect on the profession in the current context.
\end{abstract}

Key words: History of nursing, nursing, speech.

Fecha recepción: 10/10/13. Fecha aceptación: 10/06/14.

*Socióloga. Docente Escuela de Enfermería Universidad de Valparaíso. Valparaíso, Chile. Email: valentina.leal@uv.cl 


\section{INTRODUCCIÓN}

En mis trabajos sobre la enfermería he encontrado distintos documentos antiguos, algunos de ellos interesantes por sus títulos provocadores y por la característica de ser ejemplificadores de una época vivida, que más allá de hablar de enfermería o de una situación particular, nos muestran cómo se desarrollaba determinado momento histórico, cómo se vivía, qué cosas se pensaban y se decían. Entre estos documentos se encontró el texto "Lo que pueden estudiar las mujeres en Valparaíso" (1945?), escrito por la enfermera Gladys Peake en la ciudad de Valparaíso, Chile, siendo presidenta de la Asociación de Enfermeras Universitarias de Valparaíso. Actualmente, este documento pertenece al Consejo Regional del Colegio de Enfermeras de Chile A.G, no fue publicado. Sin duda, este documento se presenta ante nosotros como un objeto extraño y sugerente, con un título que se expone con potestad, que no sabemos en qué momento particular se difunde; que aproximadamente se escribe en el año 1945; que se dirige a los padres de futuras enfermeras y que realiza un trabajo argumentativo para la valoración de la profesión de enfermera $^{2}$.

En este artículo se pretende indagar en este texto, estudiándolo por medio de un análisis discursivo, bajo la perspectiva de Orlandi (1), comprendiéndolo como un objeto a situar históricamente, que responde a un periodo de la enfermería en particular, muy diferente al que se vive hoy en día. El objeto de acercarse y caracterizar la realidad pasada de la enfermería es, sin duda, para reconocerla, para reflexionar sobre los dichos de la época, identificar lo que era importante y

${ }^{1}$ Este documento fue consultado directamente desde la sede del Consejo Regional Valparaíso-San Antonio-Aconcagua del Colegio de Enfermeras de Chile A.G.

${ }^{2}$ Digo enfermera, porque el texto a analizar destaca a la enfermería como profesión femenina, en un contexto que en Chile aún no se pensaba la carrera como ámbito de desempeño para hombres. contribuir a la recuperación de la memoria histórica de enfermería. Pero también para, desde allí, pensar a la profesión en el contexto actual.

\section{LA AUTORA Y EL CONTEXTO}

Gladys Peake Guevara es la primera egresada de la Escuela Carlos Van Buren (hoy Universidad de Valparaíso) en el año 1937. Nace en Iquique un 5 de diciembre de 1910 y fallece el 1 de diciembre de 2008 en Santiago de Chile, "fue enfermera jefe del Servicio de Enfermería del Hospital Carlos Van Buren, primera directora de la Escuela de Enfermería de Concepción y enfermera jefe de la sección de Enfermería de la Dirección General del Servicio Nacional de Salud" (2). Se perfecciona en Pedagogía en enfermería y enfermería sanitaria en la Universidad de Toronto, Canadá (1942-1944), momento en que estuvo becada por la fundación Rockefeller, también obtuvo certificados de pedagogía por la misma universidad. En 1950 integra el comité de expertos de la Organización Mundial de la Salud (OMS) (3).

El texto de Gladys Peake "Lo que pueden estudiar las mujeres en Valparaíso" se escribe en un contexto de una primera etapa de la enfermería en el país, es decir, momento en que surgen las primeras carreras a nivel nacional y egresan los primeros profesionales (la misma autora es la primera egresada de enfermería en la región). Una enfermería netamente enfocada a lo femenino, sólo podían entrar mujeres "resistentes a la presión", en el caso de la Escuela Carlos Van Buren, los ingresos eran dificultosos, las postulantes eran sometidas a un riguroso proceso de selección en el cual se realizaban entrevistas personales, se evaluaba la vida de la candidata, su historia pasada y sus aptitudes de liderazgo. Así también, el régimen de la Escuela era de internado, con un control de la vida personal. La autonomía de la profesión se ejercía dentro de las posibili- 
dades de acción en dependencia al médico, en similitud a lo que ocurre en el hogar desde una visión tradicional y conservadora en que la mujer administra los recursos del hogar y el hombre provee (4).

En el contexto latinoamericano, era la época de los impulsos industrializadores y los procesos migratorios del campo a la ciudad coadyuvan al desarrollo de la atención médica, lo que impulsa el desarrollo de la industria hospitalaria entre 1940 y 1960 (5). Este periodo en la realidad nacional se caracteriza por la consolidación del frente popular al gobierno con la llegada de Pedro Aguirre Cerda en 1938. Este gobierno tuvo como particularidad el apoyo de las masas urbanas y por dar una mayor importancia a la intervención del Estado, a la industrialización y a las necesidades del trabajo (6). En materias de salud hay una preeminencia del Estado de bienestar y un sistema de salud público, que estuvo representado en el Ministerio de Salubridad, Asistencia y Previsión Social, preocupado de lo económico y sanitario, y posteriormente, por el Servicio Nacional de Salud (SNS) fundado en 1952, que coordinó prevención, fomento y recuperación, que buscaba una salud integral y cubría las acciones sanitarias y de asistencia social en un $100 \%$ y en un $70 \%$ aquellas acciones curativas hacia la población. Al SNS se debe la presencia protagónica de un equipo de salud que tuvo a su cargo las necesidades de salud y educación de la población y el establecimiento de una cultura sanitaria y eficacia epidemiológica (7). Todos estos elementos representan el contexto inmediato, las condiciones de producción en que emerge el discurso en términos sociales históricos e ideológicos en que las palabras son enunciadas y significadas.

\section{PROCEDIMIENTO DE ANÁLISIS}

El trabajo de Gladys Peake se considerará como un documento político, es por ello que lo analizaremos desde la perspectiva de los discursos, entendiendo que éstos tienen una condición en que se produce el lenguaje y un sentido que responde a relaciones sociales. El análisis de discurso, desde la perspectiva de Orlandi, es crítico, analiza las determinantes históricas del proceso de significación, es decir hay una relación entre la lengua, los sujetos que la hablan y las situaciones en que se produce el decir. Entonces el texto, como base de la unidad de análisis, porta un contexto en que se sitúan los hablantes, pero también su ideología, por ello su análisis se basa en buscar la comprensión del proceso productivo de éste. Para observar el lenguaje en sus interacciones hay que considerar tres dimensiones de argumentación: 1) relaciones de fuerza: posición del locutor y del interlocutor, 2) relación de sentido: intervinculación del discurso con "otros" y 3) relación de anticipación: previsión de la reacción de los interlocutores. El procedimiento para hacer el análisis discursivo requiere el estudio de las palabras del texto, el análisis de la construcción de las frases y la construcción de una red semántica que evidencia la dinámica entre lo social y lo gramatical (8). A esto se puede añadir: la identificación de proposiciones que se hacen y los enunciados fundamentales. En este sentido, y como en todo proceso cualitativo, se hace fundamental el rol del investigador, quien es el que interpreta.

\section{ANÁLISIS DEL TEXTO Y DEL PROCESO ARGUMENTATIVO}

"Lo que pueden estudiar las mujeres en Valparaíso"3 es un documento de 4 páginas, cuyo título demuestra el lugar desde donde se posiciona la autora que, sin duda, es desde la autoridad; el título es un imperativo "lo que pueden" (no es "propuesta de carrera

\footnotetext{
${ }^{3}$ Las citas y párrafos en cursivas han sido tomados textuales del texto.
} 
para que estudien las mujeres"), se reconoce a un hablante posicionado y conocedor de lo que habla, que se valora a sí mismo y que puede dirigirse a otro por medio de la interpelación; desde la perspectiva de Orlandi éste correspondería a un discurso autoritario, la relación de fuerza es asimétrica y con pocas posibilidades de interpretación polisémica. Esto también mantiene relación con un contexto de la enfermería, caracterizado por el liderazgo y la formación rígida de las primeras enfermeras, producto del sello bélico de la enfermería (9), como disciplina que se fortalece en contextos de guerra y que en el caso de Chile recoge toda la tradición inglesa en las primeras escuelas.

El texto comienza refiriéndose a las escasas posibilidades que tenían las jóvenes para escoger una carrera profesional en una comuna de Chile, relativamente pequeña, como es Valparaíso:

"sólo pueden optar entre el derecho y el servicio social, si sus padres no desean o no pueden enviarlas a Santiago a seguir otra carrera. Con toda razón se arguye que de estas dos profesiones, la primera ofrece pocas oportunidades para una mujer y la segunda tiene posibilidades limitadas en cuanto a obtener una buena colocación. Muchas soluciones se plantean que indudablemente ampliarían el campo de selección. Sin embargo, a pesar del problema que se presenta a los padres que desean dar a sus hijas una carrera para asegurarles un porvenir, no se hace ninguna mención de una escuela universitaria que es modelo en su género y que forma profesionales indispensables para la vida nacional”.

Como observamos en el texto, los interlocutores son en apariencia los padres y entre esas "amplias posibilidades" de escoger una carrera profesional, la autora del texto comienza a plantear un proceso argumentativo para la valoración de la enfermería ypara diferenciarla por sobre otras carreras, por ello invoca a un reconocimiento hacia la profesión de enfermera y no sólo a ésta, sino tam- bién a la casa de estudios, es decir a la Escuela Carlos Van Buren que desde 1933 comenzó a impartir la carrera.

"No debiera ser necesario en los tiempos actuales, subrayar la importancia capital que tiene la profesión para la vida y el progreso de cualquier nación consciente de lo que significa para su porvenir la salud de sus habitantes".

Al parecer existía una invisibilización de la carrera ya que no estaba en el imaginario de la educación formal, en esa dirección, el discurso subalterno de Gladys Peake apunta al reconocimiento, se presenta como un llamado a un otro para apreciar y considerar a la enfermería, más que a ser un llamado a los padres, como indica el discurso manifiesto ¿Por qué hacer un llamado para valorar la enfermería? Sin duda, porque en este periodo en Chile enfermería es una disciplina incipiente y más aún en Valparaíso, donde sólo llevaba 10 años de vida profesional. Así, este documento es, de alguna manera, un instrumento publicitario, de difusión de la enfermería y de promoción a su valorización.

Junto con el hecho de mostrar a la sociedad lo que era enfermería, también había que posicionarla en un campo laboral determinado. Promover la carrera a las mujeres, a señoritas de buen vivir. En ese sentido, la configuración de la visión de la enfermería se realiza desde el género: se apela a una profesión eminentemente femenina, donde la mujer es el elemento identitario: "Debiera pensarse en la enfermería que es la profesión femenina por excelencia". Sustentada en los valores femeninos tales como los cuidados a una gran cantidad de enfermos desvalidos, aquel no era un contexto de educación y prevención, sino una práctica curativa, de emergencia en materia de salubridad. Por eso se habla de salud=necesidad o mejor dicho enfermería=necesidad; era un periodo en que había que entrar a trabajar en un medio de epidemias, con insuficiencias inmediatas, que buscaba comenzar a educar a 
la población y allí, efectivamente, la enfermera cumplía un fuerte rol en los servicios. Aquí, la historia personal del hablante, de la visión de su profesión, se funde con el contexto: "De la historia personal se transitará a la historia social, en el sentido de la sociedad como verdadera protagonista de la atención" (10). Por eso la enfermera, se posiciona con un discurso de fuerte autovaloración, se observan a sí mismas como necesarias para su contexto, como "actividad indispensable en toda la nación y pueblo (11), expresiones que sin duda podrían seguir manifestándose pero que adquieren una significancia mayor por el contexto en que se enuncian. La enfermera se presenta como una heroína frente a una realidad adversa y a la que los otros tienen que reconocer.

Ahora bien, aparecen dos argumentos fundamentales, primeramente uno para poner en valor a la enfermería como carrera profesional, con un campo propio y un enfoque de género definido y otro para afirmar la indispensabilidad de la enfermería en el contexto actual. Pese a la autoridad en el decir que se evidencia en la autora, ella sostiene este razonamiento por una relación de sentido, vinculando su discurso a otros y apelando a la autoridad de éstos:

"Ningún programa de salud pública puede ser llevado a la práctica sin el concurso de las enfermeras... Esta es una declaración que fue sostenida por un hombre que puso el bienestar de su país en primer plano... El presidente Roosevelt... reservó un extenso párrafo al grave problema de la escasez de enfermeras y exhortó a las jóvenes mujeres a abrazar esta carrera al servicio de la comunidad".

"Ahora el presidente Truman también hace un llamado a la juventud femenina americana en el mismo sentido... no en vano estos sabios gobernantes persiguen la defensa de la salud de los habitantes, que constituyen el capital humano con que cuenta la nación".
Observamos allí la referencialidad a dos presidentes estadounidenses y el uso de un argumento basado en la falacia de la autoridad ¿por qué a ellos?, podríamos preguntarnos, si al menos Truman no tuvo un rol protector de la salud por la autorización del uso de bombas atómicas contra Japón, aunque tal vez por eso: para atender a los heridos de la guerra necesitaría profesionales especializados para sus cuidados. Avanzando un poco más en el texto podemos encontrar algunos aspectos para dilucidarlo.

"En Chile, también se están desarrollando programas modernos de salubridad auspiciados por instituciones tales como la fundación Rockefeller".

"Instituciones extranjeras conceden de preferencia becas para que las enfermeras puedan ir a estudiar a otros países donde la salubridad ha alcanzado un nivel ignorado por nosotros. Gracias a la generosidad de la fundación Rockefeller y de otras instituciones sucesivos grupos de nuestras enfermeras van cada año a los Estados Unidos y Canadá a perfeccionar sus conocimientos".

En ese entonces existía un vínculo con instituciones extranjeras que promovían el desarrollo de la profesión por medio de becas o financiamiento a programas. No sólo la salud en Latinoamérica se vio beneficiada con el aporte de organizaciones como la fundación Rockefeller, también otros países (12) y muchos reconocieron sus colaboraciones. Peake también tuvo retribución personal al ir con una beca a estudiar al extranjero fuera. Así, el discurso es coherente al mencionar y, prácticamente, agradecer a quienes colaboraron con el desarrollo de la enfermería y a quienes la valoraron y ayudaron a consolidar. Mencionar a presidentes estadounidenses es reconocer la aportación de ese país y también hacerse parte de sus proyectos pero también posiciona un discurso masculino que está en una relación de fuerza superior al de ella, que 
se presenta como el discurso del padre. Por otro lado, para Gladys Peake es importante mencionar las posibilidades de posgrado, ya que si bien enfermería tenía colocaciones en los puestos de trabajo, éstas eran mal pagadas y las opciones de posgrado se plantean como alternativa para un puesto mejor en el campo docente y de paso para contribuir al incipiente desarrollo que tenía la enfermería en el país. Es un discurso netamente estratégico.

Observamos un discurso que quiere captar a un interlocutor que se convenza, que reaccione de la ineludible necesidad de la profesión enfermera: "Tanto en la guerra como en la paz prima siempre la batalla en contra de la enfermedad" y para que puedan entender por medio de esas expresiones que hay una solución existente, una sola persona puede cumplir un rol: "Papel que solamente ella puede desempeñar". El habla apela a definir un rol propio de la enfermería, y en específico de la enfermera como mujer, que reafirma una identidad como cuidadora y doméstica, reafirmada en el proceso de embarazo y crianza que destaca una existencia de "ser en sí" y no de "ser para sí" (13) sin cuestionar siquiera si la promoción de este rol eminentemente femenino le haría bien a la profesión y contribuiría a su desarrollo y autonomía profesional.

\section{CONSIDERACIONES FINALES}

El texto de Peake apela al reconocimiento que la enfermería buscaba, al posicionamiento que requería frente a otros u otras carreras con mayor tradición, en un contexto de emergencia de la profesión y de la salud pública.

Es un discurso autoritario, que busca decir "esta es la enfermería, que sus hijas estudien, que la sociedad nos reconozca", la reacción esperada es la siguiente:
"Ahora le corresponde a la opinión pública tomarle el peso a lo que significa para nuestro país la escasez de enfermeras universitarias... hablar pues de escasez de oportunidades para la educación superior de la mujer en nuestra sociedad y no mencionar a la carrera de enfermería es desconocer el problema en su integridad y restarle una valiosa solución".

Por eso recurrió en la elaboración discursiva a los dichos de la autoridad para relevar la importancia de la enfermería y a la distinción de género como plus para dar a conocer una carrera a los padres: una carrera de señoritas, en un internado dirigido por instructoras estrictas. En el análisis del documento se desarrolla una compleja argumentación para el reconocimiento, pero también para delimitar el campo de la enfermería en un terreno determinado, es discurso político que coadyuva a definir el rol, a posicionar a la enfermería como profesión y a reafirmar su especificidad de género. Creemos que esa arma de difusión es lo que históricamente la dañaría como profesión y sería la principal traba para su desarrollo y para la consecución de una mayor autonomía respecto de la profesión médica. Pese a ello, de todos modos abre un campo femenino en un contexto de incipiente emancipación, profesionalización y de trabajo de las mujeres. Se presenta a la vez como una oportunidad para un avance, para salir del hogar, con una ligazón todavía a esa cotidianidad.

El rescate de un documento como este permite valorar el esfuerzo que algunas profesionales como Gladys Peake tuvieron que hacer para posicionar su profesión y hacerse un lugar en esta sociedad (y hacer un lugar para las mujeres). La construcción de una profesión es un proceso de largo aliento, que en ocasiones hay que recordar para valorar lo que se ha logrado y para plantearse ante los desafíos pendientes. Hoy, no es problema el valor o la necesidad de la enfermería, la 
carrera está bien posicionada tiene reconocimiento económico, los cupos de escuelas y carreras se completan y aún existen suficientes puestos de trabajo, aunque tal vez no esté resuelto el tema del reconocimiento social. Sin duda, el discurso actual es otro, el argumento en el decir también, como sus interlocutores y los medios de transmisión. Hoy, ¿por qué motivos tendría la enfermería y sus representantes apelar a los otros? ¿Qué tendría qué decir la enfermería? ¿Qué se quiere hoy, qué necesita la enfermería? ¿Mejora en sus condiciones actuales? ¿Cómo la enfermería construye y defiende su rol en la actualidad? ¿A quién necesita dirigirse y hablar? ¿A los otros, o a ellos y ellas mismas/os como enfermeras/os? ¿Qué requiere decirse y decir, cómo se plantea para elaborar un discurso para reafirmarse y defenderse, con qué medios lo hace? etc. Son cuestiones que planteo al finalizar esta reflexión.

\section{REFERENCIAS}

1. Orlandi E. Análisis del Discurso, Principios y procedimientos. Santiago, Chile: LOM ediciones/UMCE; 2012. 106 p.

2. Monardes G, Bettancourt-Ortega L, Lazcano M, Núñez N, Peroni S. Escuela de Enfermería 75 años formando profesionales al cuidado de la comunidad. Valparaíso, Chile: Universidad de Valparaíso; 2010.

3. Diccionario biográfico de Chile. $18^{\mathrm{a}}$ edición. Santiago: Editores empresa periodística Chile; 1984-1986.

4. Chuaqui-Kettlun JR, Bettancourt-Ortega LP, Leal-Román VJ, Aguirre-González CA. La identidad profesional de la enfer- mería: un análisis cualitativo de la enfermería en Valparaíso (1933-2010). Aquichán. 2014; 14(1): 53-66.

5. Verderese O. Análisis de la enfermería en la América Latina. Edu Méd Salud. 1979; 13(4): 315-339.

6. Leslie B, editor.Historia de América Latina 15. El cono sur desde 1930. Barcelona: Editorial crítica; 2002. 378 p.

7. Urriola R, Massardo J, Molina B, Monasterio $\mathrm{H}$. Historia de la protección social de la salud en Chile. Santiago: LOM ediciones/FONASA; 2009.98 p.

8. De Souza Minayo MC. La Artesanía de la investigación cualitativa. Buenos Aires: Lugar Editorial; 2013.

9. Castrillón MC. La Dimensión Social de la Práctica de la Enfermería. Historia y sociología de la enfermería. Alemania: Editorial académica española; 2012.334 p.

10. García I, Gozalbes E. Surgimiento y desarrollo de la Historia de la Enfermería en España. Enf Global. 2013; 12(30): 304310.

11. Flores R. La historia de la enfermería, síntesis de su evolución educacional de la enfermera. Santiago, Chile: Universidad de Chile; 1965.

12. Rodríguez E. La intervención de la Fundación Rockefeller en la creación de la sanidad contemporánea en España. Rev. Esp. Salud Pública [Internet]. 2000 Ene [citado 2014 Ago 18]; 74: 27-34. Disponible en: http://dx.doi.org/10.1590/S113557272000000600003

13. Siles J. La eterna guerra de la identidad enfermera: un enfoque dialéctico y decontruccionista. IndexEnferm [Internet] 2005 [citado 2014 Ago 18]; 50: 7-9. Disponible en: http://www.index-f.com/index-enfermeria/50revista/5196.php 